\title{
The Value of Positive Pressure Ventilations for Clients in Acute Respiratory Distress as a Result of Cardiac and Pulmonary Issues
}

\author{
Patrick O'Connell, RN \\ Adelphi University, Garden City, USA \\ Email: patrickoconnell@mail.adelphi.edu
}

Received 23 March 2015; accepted 24 April 2015; published 28 April 2015

Copyright (C) 2015 by author and Scientific Research Publishing Inc.

This work is licensed under the Creative Commons Attribution International License (CC BY). http://creativecommons.org/licenses/by/4.0/

(c) (7) Open Access

\begin{abstract}
Objective: Research was conducted to examine benefits to using non-invasive ventilation (NIV) or continuous positive airway pressure (CPAP) early in the treatment of respiratory distress caused by pulmonary edema, chronic obstructive pulmonary disease (COPD) and asthma. Limitations to successful NIV and CPAP therapy were evaluated to determine how prolonged initiation of treatment may lead to hypoxemia (decreased oxygen in the blood) and hypercapnia (increased carbon dioxide in the blood) resulting in poor outcomes. Method: Reviews of literature from nursing and allied health data bases (CINAHL and ProQuest) with terms pulmonary edema, positive pressure device and non-invasive ventilation from 2010 to 2014 were used. Studies were conducted in the hospital and prehospital settings. Results: The literature search located 7 articles from CINAHL and 25 articles from ProQuest. A total of 6 of these articles were analyzed. Additional sources of data were obtained from Ignatavicius and Workman (2013) Medical-Surgical Nursing PatientCentered Collaborative Care 7 th edition and American Journal of Nursing (02/2013) Volume 113: 2. Conclusion: All of the articles concluded that early initiation of continuous positive airway pressure ventilations in the short-term was beneficial; however, late initiation of therapy required additional interventions. The studies indicated that early use of positive airway pressure in acute respiratory distress improved breath rate, heart rate and blood pressure. The use of positive airway pressure for respiratory distress may decrease the need for endotracheal intubation.
\end{abstract}

\section{Keywords}

Continuous Positive Airway Pressure, Non-Invasive Ventilation, Acute Pulmonary Edema, Asthma, Chronic Obstructive Pulmonary Disease, Prehospital, Hospital 


\section{Introduction}

Respiratory distress is a major concern in the elderly and contributes to chronic disability. Adult respiratory distress syndrome (ARDS) affects 150,000 to 200,000 people in North America annually [1]. Chronic obstructive pulmonary disease (COPD) is the third leading cause of morbidity and mortality in the United States [2]. COPD is identified as narrowed airways, leading to tachypnea and accessory muscle use. The implementation of continuous positive airway pressure (CPAP) therapy to treat COPD, acute pulmonary edema (APE) and asthma has been successful as an alternative to intubation [3]. An international conference in 2012 revealed that $20 \%$ of COPD patients received NIV over intubation in 2010 [4].

CPAP is used to prevent atelectasis (collapsed alveoli) by maintaining a constant pressure during inspiration and expiration. The inspiratory phase receives pressure support (PS) to assist oxygen transfer. Positive end expiratory pressure (PEEP) is the pressure to keep the lungs partially inflated during expiration. The settings are often set at $5-15 \mathrm{~cm} \mathrm{H}_{2} \mathrm{O}$ for both settings of ventilator support. It is important that the client and caretakers have knowledge about the equipment, technique and complications of CPAP because PEEP is associated with tension pneumothorax [3]. If noninvasive ventilator management fails, clinicians need to acknowledge and implement endotracheal intubation and mechanical ventilation management quickly [2].

CPAP can be beneficial to manage dyspnea (shortness of breath), mild hypercapnia (elevated carbon dioxide in the blood) and mild hypoxia (decreased tissue oxygenation) due to exacerbations of COPD, asthma and cardiogenic pulmonary edema. CPAP decreases the complications associated with intubation including ventilatorassociated pneumonia (VAP) and failure to wean [3]. The purpose of this meta-analysis is to identify the benefits and limitations for using non-invasive ventilation (NIV) or CPAP therapy for the treatment of respiratory difficulty associated with COPD, asthma and APE.

\section{Benefits of Using NIV or CPAP}

The use of early CPAP therapy improves blood oxygenation and blood gases by preventing atelectasis and pulmonary shunting [5]. Myocardial damage is lessened according to results of serum creatinine kinase and serum aspirate aminotransferase activity. Heart rate, blood pressure, respiratory rate and blood oxygen saturation improves with the use of CPAP therapy [6]. Studies showed a significant decrease of in-hospital mortality among patients whose pulmonary edema was caused by myocardial infarction (MI) or ischemia [7]. The incidence of new MI's was unchanged with the use of CPAP alone however; there was benefit with the addition of standard therapy (oxygen, nitrates, morphine, diuretics and supportive care). The four general rules for guiding care for COPD are addressing precipitating factors, reducing air trapping, decreasing pulmonary inflammation and improving gas exchange [2]. Evidence shows that $70 \%$ of patient's receiving NIV for cardiogenic pulmonary edema demonstrated a significant reduction of endotracheal intubation [8]. In the prehospital setting, CPAP therapy is associated with short-term mortality [5]. NIV is recommended for the treatment of moderate to severe respiratory acidosis (pH, 7.25 to 7.35 ) or hypercapnia less than $45 \mathrm{mmHg}$ [2]. $\mathrm{pH}$ values that indicate levels of success using CPAP or NIV therapy are detailed in Diagram 1. NIV decreases tachypnea, dyspnea, respiratory muscle fatigue and respiratory acidosis.

\section{Limitations of Using NIV or CPAP}

Low use of NIV in some hospitals has to do with lack of knowledge, confidence, experience and funding. NIV is not advisable for hemodynamic compromised patients [4]. With the growing shortage of ICU beds in hospitals, options are limited to either delaying NIV or initiating NIV therapy on the ward. Prolonging therapy increases blood carbon dioxide levels and decreases successful CPAP therapy [9]. There was no difference in intensive care unit (ICU) mortality or in hospital mortality for several trials due to NIV patients in need of intubation [8]. Recommendations for NIV in addition to standard care are suggested for COPD patients with respiratory acidosis ( $\mathrm{pH}$ less than 7.35). A reduced need for intubation was identified with the use of CPAP in the presence of pulmonary edema however; the incidence of new MI's and mortality was unchanged compared to standard therapy [7]. Some patients in the study might have benefited from lower or higher PEEP settings [6]. Endotracheal intubation is indicated for clients who have respiratory arrest, respiratory pauses, ventricular arrhythmias, impaired mental status, history of massive aspiration, unable to move secretions, severe hypoxemia, clients unable to tolerate NIV or unchanged arterial blood gases (ABG's) within 30 minutes of NIV initiation [2]. 
$\mathrm{PH}$ values that indicate levels of success using CPAP therapy

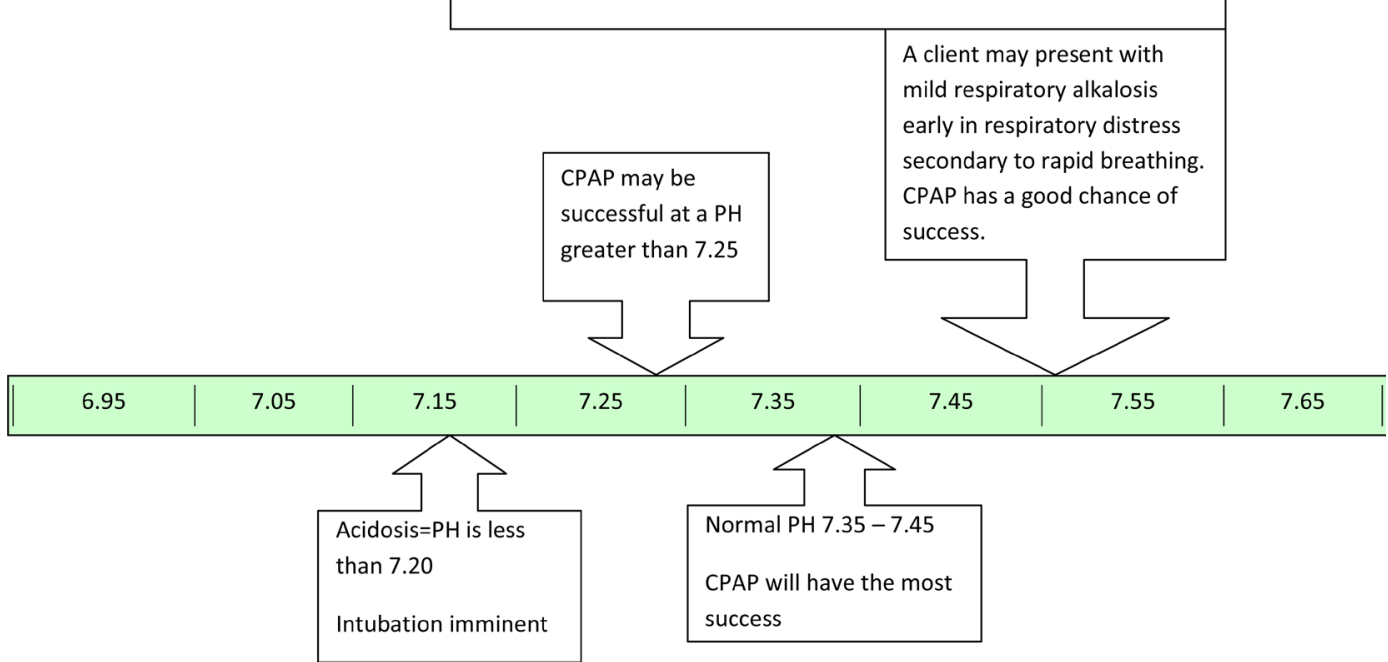

Diagram 1. pH values are acidic to the left and alkaline to the right on the diagram. The normal $\mathrm{pH}$ value of the human body is 7.35 to 7.45 . CPAP therapy success declines as $\mathrm{pH}$ moves to the left of normal values.

\section{Results of Literature}

\subsection{Prehospital}

A study including 387 patients in which 149 had early CPAP therapy introduced. Results showed that oxygen saturation (Sao2) increased from 77\% to 90\%, pulse rate decreased from 106 to 100 beats per minute and systolic and diastolic blood pressure parameters stabilized [10]. A meta-analysis using 12 articles from electronic medical databases (Cochrane, Medline, EMBASE and CINAHL) was conducted. Results suggested that the use of CPAP in the prehospital setting is beneficial for patients with pulmonary edema [5]. A meta-analysis was conducted using 33 articles which showed evidence that supports using NIV for patients with pulmonary edema secondary to acute myocardial ischemia or infarct [7]. A study using 340 patients from 2 emergency ambulance companies over a six month perspective was conducted. Their results showed 56 patients discontinued CPAP therapy due to anxiety, need for intubation, apnea, mask problems and improvement. The patients that remained on CPAP therapy demonstrated 241 improved, 51 stayed the same and 45 worsened. The study showed that lowfractional oxygen (28\% - 30\%) CPAP is beneficial for acute respiratory distress. Low-fractional oxygen is safe for patient with COPD and does not interfere with their hypoxic drive. The devices were easy to use and cost effective. Improvements in respiratory rates, blood oxygen saturations and subjective data were seen with $71.5 \%$ of the patients treated [6].

\subsection{Hospital}

A lecture was presented for the American Association for Respiratory Care. It identified that CPAP is beneficial in the early stages of COPD, APE and asthma however; severely acidotic clients may have poor outcomes. Endotracheal intubation has adverse effects related to VAP and higher mortality [4]. A study of 1123 Medical Emergency Team (MET) calls on a ward in which 129 patients were analyzed. Fifty-four patients were in an NIV group and 75 patients were in the no-NIV group. The NIV groups had lower arterial blood pH, higher bicarbonate values and had hypercapnia. The NIV group outcomes showed more improvement possibly due to more time spent with them during the MET call. Results suggested that NIV can be safely started in the context of a MET evaluation for COPD, asthma and APE. In the absence of close monitoring, the NIV group had lower rates of ICU transfers and need for intubation [9]. A meta-analysis using randomized control trials (RCT's) from electronic medical databases (MEDLINE, EMBASE, CINAHL, CENTRAL, DARE, Cochrane, ACP Journal Club, the metaRegistry of Controlled Trials, ClinicalTrials.gov and the Journals@Ovid) was conducted. Sixteen 
members of the Canadian Critical Care Society in eight pairs evaluated the data to develop guidelines for the use of CPAP or NIV in the acute care setting and to identify endotracheal intubation and hospital mortality outcomes. Results suggested NIV is an important option for patients at risk of respiratory failure in the acute care setting. The use of NIV or CPAP is recommended in patients with pulmonary edema in the absence of shock or acute coronary syndrome (ACS). The use of CPAP for exacerbation of COPD and asthma had no recommendation due to lack of RCT's. NIV for acute lung injury is not recommended. Evidence based clinical recommendations were made to optimize NIV use [8].

\subsection{Limitations to Research}

Studies were limited to the paramedic's interpretation and there was discordance in patient enrollment between the two emergency ambulance systems. Transport times and altitude may have accounted for differences seen [6]. The need for future research for the use of NIV for severe community-acquired pneumonia, asthma, acute lung injury and chest trauma was suggested [5]. Medications used, small size trials, pre-existing illnesses, alterations in pressure support and level of distress limited study results [7]. The lack of high quality studies, transport time and unsuccessful field intubation limited the research [5].

\section{Conclusion}

All of the literature review supported evidence of improved patient outcomes with early initiation of CPAP therapy. Vital signs stabilized and myocardial damage lessened with early NIV or CPAP use. It was mentioned that some clients may have had better outcomes with the addition of standard therapy or changes of PEEP settings. Prolonged initiation of NIV or CPAP therapy revealed increased acidosis resulting in poor outcomes. Several studies were limited to their research due to lack of large or high quality RCT's. Recommendations for future trials of CPAP therapy for community-acquired infections, lung injury, chest trauma and asthma were advised. This meta-analysis explores benefits and limitations for the use of NIV or CPAP therapy in the prehospital and hospital settings. This information is intended for prehospital and hospital organizations to develop and implement guidelines for NIV or CPAP therapy in the presence of respiratory distress as a result of cardiac and pulmonary issues.

\section{Acknowledgements}

The author is grateful to Professor Kathryn Lang, MSN, Adelphi University for her support and expert advice regarding scholarly writing.

\section{References}

[1] ARDS Foundation (2010) Facts about ARDS, ARDS Foundation. www.ardsil.com/facts.htm

[2] Burt, L. and Corbridge, S. (2013) COPD Exacerbations. American Journal of Nursing, 113, 34-44.

[3] Ignatavicius, D. and Workman, L. (2013) Medical-Surgical Nursing: Patient-Centered Collaborative Care. 7th Edition, Elsevier Inc., St. Louis.

[4] Nava, S. (2013) Behind a Mask: Tricks, Pitfalls, and Prejudices for Noninvasive Ventilation. Respiratory Care, 58, 1367-1376.

[5] Williams, B., Boyle, M., Robertson, N. and Giddings, C. (2012) When Pressure Is Positive: A Literature Review of the Prehospital Use of Continuous Positive Airway Pressure. Prehospital and Disaster Medicine, 28, 52-60.

[6] Bledsoe, B., Anderson, E., Hodnick, R., Johnson, L., Johnson, S. and Dievendorf, E. (2012) Low-Fractional Oxygen Concentration Continuous Positive Airway Pressure Is Effective in the Prehospital Setting. Prehospital Emergency Care, 16, 217-221.

[7] Weng, C., Zhao, Y., Liu, Q., Fu, C., Sun, F., Ma, Y. and He, Q. (2010) Meta-Analysis: Noninvasive Ventilation in Acute Cardiogenic Pulmonary Edema. Annals of Internal Medicine, 152, 590-600. http://dx.doi.org/10.7326/0003-4819-152-9-201005040-00009

[8] Keenan, S., Sinuff, T., Burns, K., Muscedere, J., Kutsogiannis, J., Mehta, S., Dodek, P., et al. (2011) Clinical Practice Guidelines for the Use of Noninvasive Positive-Pressure Ventilation and Noninvasive Continuous Positive Airway Pressure in the Acute Care Setting. Canadian Medical Association Journal (CMAJ), 183, E195-E214. http://dx.doi.org/10.1503/cmaj.100071 
[9] Khalid, I., Sherbini, N., Qushmaq, I., Qubajah, M., Nisar, A., Khalid, T. and Hamad, W. (2014) Outcomes of Patients Treated with Noninvasive Ventilation by a Medical Emergency Team on the Wards. Respiratory Care, 59, 186-192. http://dx.doi.org/10.4187/respcare.02515

[10] Dib, J., Martin, S. and Luckert, A. (2012) Prehospital Use of Continuous Positive Airway Pressure for Acute Severe Congestive Heart Failure. Journal of Emergency Medicine, EMJ, 42, 553-558. http://dx.doi.org/10.1016/j.jemermed.2011.06.002 